\title{
The Calibration Kit Spectral Fluorescence Standards-A Simple and Certified Tool for the Standardization of the Spectral Characteristics of Fluorescence Instruments
}

\author{
D. Pfeifer • K. Hoffmann - A. Hoffmann - C. Monte • \\ U. Resch-Genger
}

Received: 7 November 2005 / Accepted: 21 February 2006 / Published online: 22 June 2006

(C) Springer Science+Business Media, Inc. 2006

\begin{abstract}
With the Calibration Kit Spectral Fluorescence Standards BAM-F001-BAM-F005, we developed a simple tool for the characterization of the relative spectral responsivity and the long-term stability of the emission channel of fluorescence instruments under routine measurement conditions thereby providing the basis for an improved comparability of fluorescence measurements and eventually standardization. This first set of traceable fluorescence standards, which links fluorescence measurements to the spectral radiance scale in the spectral range of 300-770 $\mathrm{nm}$ and has been optimized for spectrofluorometers, can be employed for different measurement geometries and can be adapted to different fluorescence techniques with proper consideration of the underlying measurement principles.
\end{abstract}

Keywords Fluorescence - Standard - Spectral correction · Emission - Calibration tool

\section{Introduction}

For luminescence techniques that are widely used in life and material sciences as well as in environmental analysis [15], there is an increasing need for reliable and comparable data, quantification, and eventually standardization having applications in strongly regulated areas like e.g., medical

D. Pfeifer · K. Hoffmann · A. Hoffmann · C. Monte .

U. Resch-Genger

Federal Institute for Materials Research and Testing, Working

Group Optical Spectroscopy, Division I.5,

Berlin, Germany

U. Resch-Genger $(\square)$

e-mail: ute.resch@bam.de diagnostics at reach. To rule out instrumentation as a major source of variability and to improve the comparability of fluorescence measurements across instruments and over time, fit-for-purpose standards in combination with tested protocols for instrument characterization and performance validation (IPV) are required [6]. As a first step towards simple and multifunctional calibration tools for fluorescence instruments that link luminescence measurements to radiometric units [7], we developed the Calibration Kit Spectral Fluorescence Standards BAM-F001-BAM-F005 including the software LINKCORR [8]. This first set of traceable fluorescence standards enables the determination of the relative spectral responsivity $s(\lambda)$ and the long-term stability of the emission channel of fluorescence instruments in the spectral range of 300-770 $\mathrm{nm}$ thereby also providing the basis for spectrally corrected, i.e., instrument-independent emission spectra. This calibration tool that was recently tested in an interlaboratory comparison of the National Metrological Institutes NIST, NRC, PTB, and BAM [8] was certified by us in January 2006 [9]. Here, the working principle of BAM-F001-BAM-F005 and the criteria for the design of this Calibration Kit are presented as well as application-relevant properties of the kit components investigated by us. ${ }^{1}$

\section{Experimental}

Solvents and reagents

The Calibration Kit Spectral Fluorescence Standards BAMF001, BAM-F002, BAM-F003, BAM-F004, and BAM-F005

\footnotetext{
${ }^{1}$ NIST: National Institute of Standards and Technology, USA; NRC: National Research Council, Canada, PTB: Physikalisch-Technische Bundesanstalt, Germany; BAM: Federal Institute for Materials Research and Testing, Germany.
} 
are available from BAM as well as through all subsidiaries of Sigma-Aldrich with Fluka product numbers 72594, 23923, 96158,74245 , and 94053 , respectively, and 97003-1KT-F for the kit. This kit includes the five bottled dyes BAM-F001BAM-F005 ready-made by Sigma-Aldrich GmbH (former Fluka Production $\mathrm{GmbH}$ ), the solvent ethanol, and a BAM certificate of the normalized corrected emission spectra of the kit components and the corresponding wavelengthdependent expanded relative uncertainties of the certified fluorescence intensities [9]. Included are also the software LINKCORR for data evaluation, and a standard operation procedure (SOP) for the use of the kit and of LINKCORR. The working principle of LINKCORR that was developed by BAM for the calculation of the (inverse) relative spectral responsivity of fluorescence instruments from the BAMcertified corrected emission spectra of the kit dyes and their uncorrected fluorescence spectra measured with the instrument to be calibrated is documented in the SOP supplied with the kit. LINKCORR has been successfully tested with different fluorescence instruments and different operating systems as well as by different operators/users. ${ }^{2}$

The dyes X, Y, and quinine sulfate dihydrate (QS; SRM 936a, NIST) used for the control of a kit dye-based emission correction curve and accordingly corrected fluorescenceemission spectra are of the highest purity available. They were obtained from Sigma-Aldrich $\mathrm{GmbH}$ and the National Institute of Standards and Technology (NIST). The solvents ethanol, acetonitrile, and perchloric acid used for dye X, Y, and QS, respectively, were of the highest purity available and were purchased from Sigma-Aldrich GmbH (ethanol; acetonitrile) and Merck.

\section{Equipment}

Absorption spectra were recorded on a CARY 5000 spectrophotometer from Varian Inc. The corrected emission spectra of BAM-F001-BAM-F005 were obtained with a previously described Spectronics Instruments 8100 spectrofluorometer [8]. The wavelength- and polarization-dependent spectral responsivity $s(\lambda)$ of this fluorometer was determined with an integrating sphere-type spectral radiance transfer standard (quartz halogen lamp placed inside an integrating sphere, Gigahertz-Optik GmbH; $[8,10]$ ) illuminating a nonfluorescent diffuse reflectance standard (Gigahertz Optik $\mathrm{GmbH}$ ) [8]. Both transfer standards were calibrated by the Physikalisch-Technische Bundesanstalt (PTB). A detailed description of the instrument calibration has been recently published [8].

\footnotetext{
${ }^{2}$ BAM-F001-BAM-F005: dyes A-E in earlier publications.
}

Procedures

All the absorption and emission measurements were performed at a temperature of $(25 \pm 2)^{\circ} \mathrm{C}$. Typically, dilute dye solutions with absorbances $A \leq 0.04$ at the excitation wavelength equaling to the longest wavelength absorption maximum were used [9]. The certified normalized corrected emission spectra of BAM-F001-BAM-F005 were measured in ethanol at $25^{\circ} \mathrm{C}$ with three different spectral bandpasses of the emission monochromator of the BAM fluorometer, i.e., 1,4 , and $8 \mathrm{~nm}$ [9]. Prior to correction for the relative spectral responsivity $s(\lambda)$ of the emission channel, a background spectrum $\left[I_{\mathrm{b}}\left(\lambda_{\mathrm{em}}\right)\right]$, recorded under identical measurement conditions for a blank solvent sample, was subtracted from the measured spectrum $\left[I_{\mathrm{m}}\left(\lambda_{\mathrm{em}}\right)\right]$ to remove scattering and fluorescence from the solvent and dark counts of the detector. Correction of the accordingly obtained uncorrected emission spectra $\left[I_{\mathrm{u}}\left(\lambda_{\mathrm{em}}\right)=I_{\mathrm{m}}\left(\lambda_{\mathrm{em}}\right)-I_{\mathrm{b}}\left(\lambda_{\mathrm{em}}\right)\right]$ for $s(\lambda)$ was achieved by division of $I_{\mathrm{u}}\left(\lambda_{\mathrm{em}}\right)$ by the relative spectral responsivity of the emission channel obtained with physical transfer standards (PTS) [8]. The resulting PTS-based and BAM-certified corrected fluorescence emission spectra $I_{\mathrm{c}}\left(\lambda_{\mathrm{em}}\right)$ are traceable to the spectral radiance scale [8]. PTS-based corrected emission spectra of dye X, dye Y, and QS were similarly obtained by us.

Instrument characterization with BAM-F001-BAM-F005 and LINKCORR yields always the instrument's inverse relative spectral responsivity $1 / s(\lambda)$ termed emission correction curve, as also described in the certificate of the Calibration Kit [9]. Accordingly, in the case of a kit dye-based emission correction, corrected emission spectra are obtained by multiplication of $I_{\mathrm{u}}\left(\lambda_{\mathrm{em}}\right)$ by $1 / s(\lambda)$. These spectra are traceable to the spectral radiance scale. The relative spectral responsivity of the fluorescence instruments results from the reciprocal of the output of LINKCORR, i.e., $1 / s(\lambda)$.

\section{Results and discussion}

Instrument effects on fluorescence signals

Luminescence techniques always yield signals $I_{\mathrm{u}}\left(\lambda_{\mathrm{ex}}, \lambda_{\mathrm{em}}\right)$ that contain sample- and instrument-specific contributions [6], see Eq. (1). Signal-relevant analyte properties are the chromophore's absorptance at the excitation wavelength $\alpha\left(\lambda_{\text {ex }}\right)$ and its spectral fluorescence yield $F_{\lambda}\left(\lambda_{\text {ex }}, \lambda_{\text {em }}\right)[8,10]$. Instrument-specific effects include the spectral irradiance at sample position $E_{\text {ex }, \lambda}\left(\lambda_{\text {ex }}\right)$ and the spectral responsivity of the emission channel $s\left(\lambda_{\mathrm{em}}\right){ }^{3}$ These two wavelength-, polarization-, and time-dependent quantities reflect the

\footnotetext{
${ }^{3}$ Due to radiometric convention, $s(\lambda)$ always implies $s_{\lambda}(\lambda)$.
} 


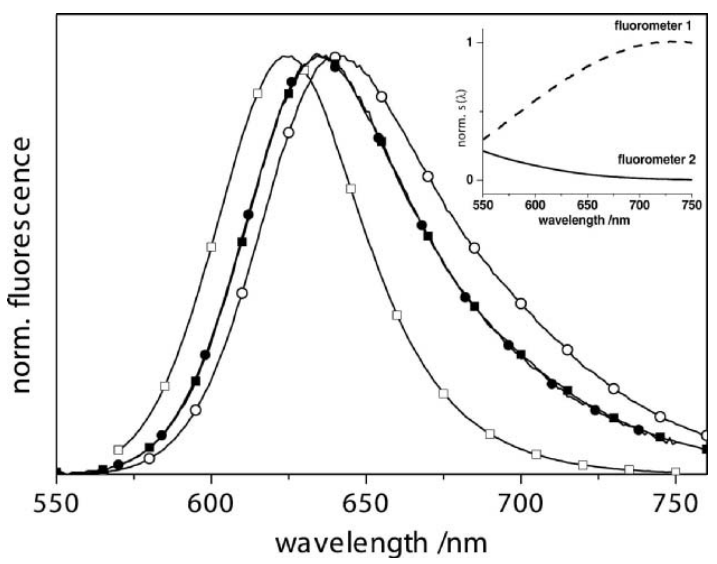

Fig. 1 Normalized uncorrected (open symbols) and corrected (full symbols) emission spectra of a typical organic chromophore measured with two different fluorometers. The normalized spectral responsivities $s(\lambda)$ of both instruments are depicted in the inset. Normalization was performed at the peak maxima

spectral radiance of the excitation light source, the transmittance of optical components like lenses, mirrors, filters, monochromator gratings, and polarizers in the excitation and emission channel, and the spectral responsivity of the detection system of the respective fluorescence instrument. The influence of $s\left(\lambda_{\mathrm{em}}\right)$ on fluorescence data and the need for its consideration, i.e., spectral (emission) correction are illustrated in Fig. 1 exemplary for a typical organic fluorophore and two spectrofluorometers.

$I_{\mathrm{u}}\left(\lambda_{\mathrm{ex}}, \lambda_{\mathrm{em}}\right)=\alpha\left(\lambda_{\mathrm{ex}}\right) F_{\lambda}\left(\lambda_{\mathrm{ex}}, \lambda_{\mathrm{em}}\right) E_{\mathrm{ex}, \lambda}\left(\lambda_{\mathrm{ex}}\right) s\left(\lambda_{\mathrm{em}}\right)$

The comparability of fluorescence data across instruments as well as e.g., the use of fluorescence spectra for the identification of analytes and the determination of fluorescence quantum yields rely on the knowledge of and the correction for $s\left(\lambda_{\mathrm{em}}\right)$ and its time-dependent changes thereby taking also aging of instrument components into account $[8,11]$. From the emission side, the determination of the instrument's relative spectral responsivity is sufficient for the elimination of instrument-specific wavelength and polarization dependences in the vast majority of cases. This is by far more easy to realize than the absolute measurement of this quantity. Consideration of time-dependent changes of $s(\lambda)$ makes its regular determination mandatory. Simultaneously, it comprises an elegant tool for the validation of the instrument performance validation and instrument long-term stability.

Determination of the spectral responsivity $s\left(\lambda_{\mathrm{em}}\right)$

$s\left(\lambda_{\text {em }}\right)$ can be obtained with a source that emits a known and broad spectrum in the UV/-vis/NIR region $[6,8,12,13,14]$. Its reliable determination as the basis for the spectral

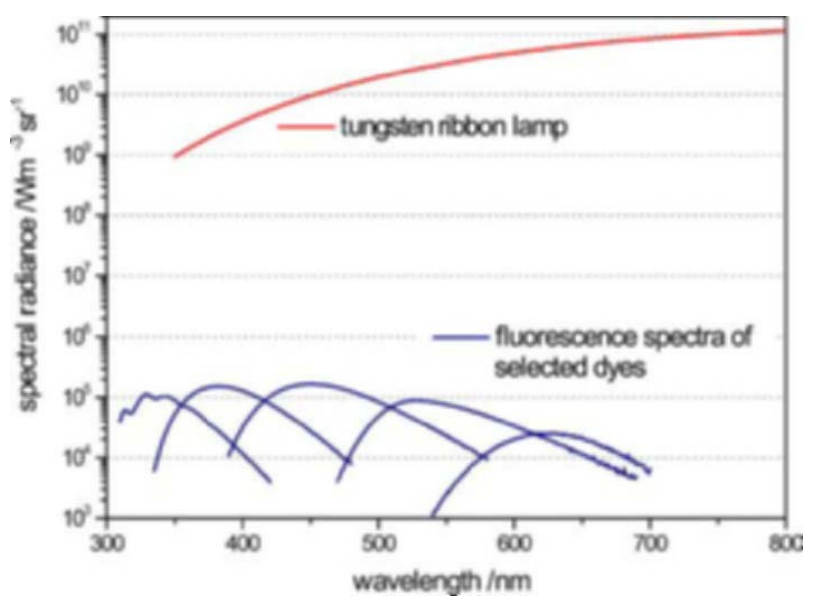

Fig. 2 Comparison of the typical spectral radiances of a tungsten ribbon lamp (top) and of typical fluorophores (bottom)

correction of emission spectra requires not only control and if necessary consideration of the wavelength accuracy of its emission channel [15] and the operation of the detection system within its linear range, but also consideration of the samples to be corrected and thus measurement conditions commonly employed [8]. This includes measurement geometry, format of the sample, and typical instrument settings. Tungsten ribbon lamps and integrating sphere-type spectral radiance transfer standards are traceable, but are tedious to align, can impose restrictions on measurement geometry, need regular and expensive recalibrations, and often do not fit into compact fluorescence instruments. Moreover, their spectral radiances or emission intensities exceed those of common fluorescent samples by approximately four (tungsten ribbon lamp) to two (integrating sphere radiator; not shown) orders of magnitude, see Fig. 2. Accordingly, their use requires attenuation procedures that do not introduce additional spectral effects. This circumvents errors due to the operation of the detection system within its nonlinear range $[8] .^{4}$

In contrast, chromophore-based spectral fluorescence standards $[6,8,12,13,14,16]$ are easy to operate. Especially liquid standards such as BAM-F001-BAM-F005 offer an unique flexibility with respect to measurement geometry, format or type of container, and type of instrument to be calibrated. Moreover, fit-for-purpose fluorescence standards can be measured under typical, i.e., routine conditions due to the close match between both their spectral radiances/luminescence intensities and the size and shape of their radiating volume with that of commonly analyzed samples. Thus, many sources of systematic error inherent to instrument calibration with physical transfer standards $[8,10]$

\footnotetext{
${ }^{4}$ In the case of ratio mode-type instruments that record the quotient of the signal of the emission and the reference channel, both detectors need to be operated within their linear range.
} 
can be elegantly circumvented. However. the-compared to spectral radiance transfer standards-narrow emission spectra of organic and inorganic chromophores require the combination of different fluorescence standards to a set to cover the UV/vis/NIR $[8,17]$. Aside from the proper choice and combination of candidate materials according to recently published criteria discussed in the following section [8], the most important prerequisites for the reliability of spectral fluorescence standards, however, are the precise and traceable measurement of their corrected emission spectra with a reported uncertainty and a properly characterized instrument and the characterization of their calibration-relevant properties according to EN ISO/IEC 17025 and ISO Guides 34 and 35 [7, 8]. To enable users of such fluorescence standards to judge their quality and reliability, in addition to the calibration-relevant properties of these reference materials also the type and design of the fluorescence instrument used for standard characterization as well as the employed calibration strategies and transfer standards need to be described.

\section{Calibration Kit Spectral Fluorescence Standards:} working principle and properties

As a first step towards standardization of the spectral characteristics of fluorescence instruments on a broad level, we developed the Kit Spectral Fluorescence Standards covering the spectral region of $300-770 \mathrm{~nm}$. This calibration tool consists of the five spectral fluorescence standards BAM-F001BAM-F005 with certified normalized corrected emission spectra, the solvent ethanol, and the software LINKCORR [9]. Its straightforward working principle is illustrated in Fig. 3. Addition of $10 \mathrm{ml}$ of ethanol to each of the kit components provided as ready-made bottled solids yields working solutions with absorbances of approximately 0.04 at the maximum of each dye's lowest energy absorption band. These solutions are measured with the instrument to be calibrated under measurement conditions for which the (inverse) relative spectral responsivity and spectrally corrected emission spectra are eventually desired. Data evaluation should be performed with the BAM-software LINKCORR provided with the kit. LINKCORR calculates the quotients $\mathrm{Q}^{\mathrm{F} 00 \mathrm{x}}\left(\lambda_{\mathrm{em}}\right)$ of the BAM-certified corrected emission spectra $\mathrm{I}_{\mathrm{c}}\left(\lambda_{\mathrm{em}}\right)$ (Fig. 3, bottom, solid lines) and the measured uncorrected emission spectra $I_{u}\left(\lambda_{e m}\right)$ (Fig. 3, bottom, dashed lines) for each dye by division of the former by the latter. The certified data measured in 1-nm data intervals are supplied on $\mathrm{CD}$ with the kit in a format readable by LINKCORR. Combination of the resulting ratios $\mathrm{Q}^{\mathrm{F} 00 \mathrm{x}}\left(\lambda_{\mathrm{em}}\right)\left(\mathrm{Q}^{\mathrm{F} 00 \mathrm{x}}\left(\lambda_{\mathrm{em}}\right)=\mathrm{I}_{\mathrm{C}}{ }^{\mathrm{F} 00 \mathrm{x}}\left(\lambda_{\mathrm{em}}\right) / \mathrm{I}_{\mathrm{U}}{ }^{\mathrm{F} 00 \mathrm{x}}\left(\lambda_{\mathrm{em}}\right)\right)$ is performed by a statistical weighting procedure implemented in LINKCORR with the resulting global emission correction curve $1 / s(\lambda)$ (Fig. 3, top) being the actual output of LINKCORR. Its reciprocal yields the relative spectral

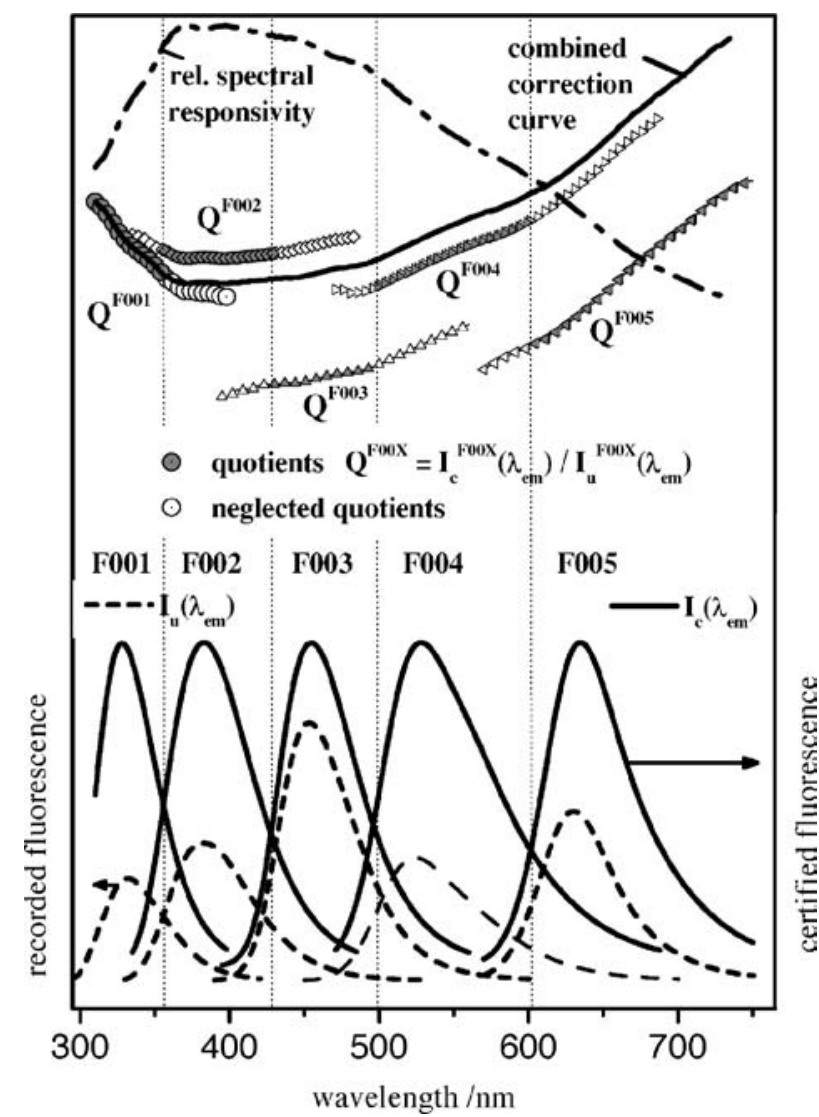

Fig. 3 Working principle of the Calibration Kit Spectral Fluorescence Standards. Division of the certified corrected emission spectra of BAMF001-BAM-F005 $\left[\mathrm{I}_{\mathrm{c}}\left(\lambda_{\mathrm{em}}\right)\right.$, bottom, solid lines $]$ by the corresponding recorded uncorrected spectra $\left[\mathrm{I}_{\mathrm{u}}\left(\lambda_{\mathrm{em}}\right)\right.$, bottom, dashed lines $]$ of these dyes as performed by the BAM software LINKCORR leads to individual quotients $\mathrm{Q}^{\mathrm{F} 00 \mathrm{x}}\left(\lambda_{\mathrm{em}}\right)=\mathrm{I}_{\mathrm{c}}{ }^{\mathrm{F} 00 \mathrm{x}}\left(\lambda_{\mathrm{em}}\right) /\left(\mathrm{I}_{\mathrm{u}}^{\mathrm{F} 00 \mathrm{x}}\left(\lambda_{\mathrm{em}}\right)\right.$, see middle part. Weighted combination of $\mathrm{Q}^{\mathrm{FO0x}}\left(\lambda_{\mathrm{em}}\right)$ by LINKCORR yields an overall emission correction curve for the chosen measurement conditions of the instrument to be calibrated, see upper part. This curve, i.e., the output of LINKCORR equals the instrumentś inverse relative spectral responsivity $1 / s\left(\lambda_{\mathrm{em}}\right)$. Its reciprocal yields the relative spectral responsivity $\mathrm{s}\left(\lambda_{\mathrm{em}}\right)$ of the respective fluorescence instrument, see also upper part. Corrected instrument-independent and thus comparable spectra are obtained by multiplication of measured spectra with the emission correction curve $1 / s\left(\lambda_{\text {em }}\right)$

responsivity of the respective fluorescence instrument (Fig. 3, top).

Corrected, i.e., instrument-independent data are obtained by multiplication of measured spectra with the emission correction curve $1 / s\left(\lambda_{\mathrm{em}}\right)$. Such corrected spectra are comparable across instruments and are traceable to the spectral $r a$ diance scale. For the determination of relative fluorescence quantum yields, accordingly corrected spectra have to be multiplied by the wavelength prior to integration on a wavelength scale, as the fluorescence quantum yield is the ratio of the number of emitted to absorbed photons and not a ratio of radiated fluxes or powers $[8,9]$. Determination of the emission correction curve at regular intervals, i.e., typically every 
6 months, as recommended by BAM [9] provides a simple tool for the control and consideration of the long-term stability of the emission channel and thus for time-independent fluorescence data.

\section{Kit properties}

The reference materials BAM-F001-BAM-F005 with the certified property corrected emission spectrum have been developed according to ISO Guides 34 and 35 and are traceable to the spectral radiance scale $[10,15]$. The provided wavelength-dependent relative uncertainties of the corrected emission spectra combine the relative uncertainties of the calibration of the BAM fluorometer used for certification and the relative uncertainties of the measurements of the emission spectra with this instrument as well as materialrelated uncertainties derived from homogeneity and stability studies according to ISO Guide 35 [9,20]. These relative uncertainties were determined by the propagation of uncertainties according to the Guide to the Expression of Uncertainty (GUM) and ISO Guide 35 [9].

The kit components were chosen according to the following criteria. (i) Broad and unstructured emission spectra minimize the dependence of the shape of the spectra on instrument spectral bandpass/instrument resolution. (ii) Moderate to strong fluorescence quantum yields increase the signal-to-noise ratios and strongly reduce the influence of stray light, solvent emission, and fluorescent impurities on the emission spectra. (iii) For the design of a standard set, the fluorescence spectra of spectrally neighboring chromophores must cross at points of sufficient fluorescence intensity, e.g., at least at $20 \%$ of the relative maximum fluorescence intensity. (iv) The minimum spectral overlap between absorption and emission of the kit components results in a very moderate influence of dye concentration and measurement geometry on the spectral shape of the emission spectra of the fluorescence standards. (v) The in most of the cases only very moderate dependence of the spectral shape of the dyes' emission spectra on excitation wavelength provides a certain flexibility of the excitation wavelengths to be used [9]. (vi) The small fluorescence anisotropy $(r)$ of the dyes, e.g. $r \leq 0.05$ within the analytically relevant room temperature region yields a virtually isotropic fluorescence [8]. This circumvents additional polarization effects and thus minimizes uncertainties for the use of the fluorescence standards under measurement conditions that can dispense with or for instruments that lack polarizers. (vii) The thermal and photochemical stability of the kit components has been tested in the solid state and in ethanolic solution under as many application-relevant conditions as possible to guarantee for a sufficient stability of the reference materials and to define the shelf life and storage conditions of the kit [9]. As described in the certificate, ready-made bottled BAM-F001,
BAM-F002, BAM-F003, BAM-F004, and BAM-F005 can be stored in the refrigerator at $4{ }^{\circ} \mathrm{C}$ for 6 months without any changes in their certified properties. Solutions of these dyes can be used for a week at maximum, if stored in the dark at $4^{\circ} \mathrm{C}$ in properly closed containers. Uptake of water has to be minimized to avoid spectral changes of the emission spectra of the kit components and thus deviations from the certified property. (viii) The purity of the kit dyes was determined by several analytical techniques such as e.g., liquid chromatography with absorption and fluorescence detection, gas chromatography, and quantitative NMR [9]. This guarantees for the reproducibility of the kit components if the material on stock is eventually consumed. (ix) With the supply of the kit solvent ethanol with BAM-F001-BAM-F005, effects of the microenvironment on the dyes' emission spectra are minimized. The fullfillment of these requirements renders the Calibration Kit Spectral Fluorescence Standards not only suitable for the reliable calibration of spectrofluorometers in $0^{\circ} / 90^{\circ}$ measurement geometry employing $1-\mathrm{cm}$ cells, but also for use in different measurement geometries and in different sample containers. Moreover, it eventually provides the basis for the adaptation of the Calibration Kit to the characterization of a broad variety of different types of fluorescence instruments ranging from spectrofluorometers over microplate readers to spectral imaging systems [18].

Evaluation of the calibration kit spectral fluorescence standards in interlaboratory comparisons

Both the corrected emission spectra of BAM-F001-BAMF005 and the determination of a dye-based emissioncorrection curve with the BAM kit and LINKCORR have been evaluated and confirmed in an interlaboratory comparison of the National Metrological Institutes NIST, NRC, PTB, and BAM [9,19, mauscript in preparation]. This comparison included the measurement of the corrected emission spectra of the dyes in two measurement geometries, i.e., $0^{\circ} / 90^{\circ}$ and $45^{\circ} / 0^{\circ}$ and at two different concentrations with four fluorescence instruments of different design that were all traceably calibrated with different physical transfer standards (PTS) employing source- and detector-based calibration schemes. In a second step, the corrected emission spectra of the three test dyes X, QS, and Y obtained with a PTS- and a kit dye-based spectral correction were compared. In all cases, the agreement of the spectra was excellent [19].

Moreover, this calibration tool has been successfully tested with several types of common spectrofluorometers by selected users from industry and academics. Exemplary chosen results are depicted in Fig. 4 that compares the uncorrected and kit dye-based corrected emission spectra of the dyes $\mathrm{X}, \mathrm{QS}$, and $\mathrm{Y}$ measured with four typical fluorometers. As follows from Fig. 4, the comparability of the kit 

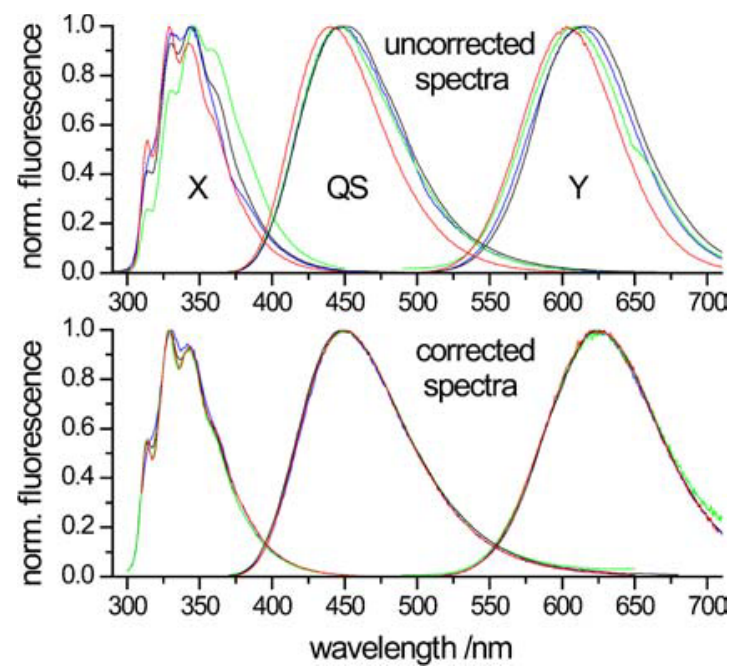

Fig. 4 Evaluation of the Calibration Kit Spectral Fluorescence Standards with common spectrofluorometers. Kit dye-based spectral correction of the uncorrected emission spectra of the test dyes X, QS, and $\mathrm{Y}(t o p)$ measured with four different fluorometers and comparison of the resulting corrected emission spectra (bottom). A comparability of the corrected spectra better than $10 \%$ is achieved

dye-based corrected fluorescence spectra is better than $10 \%$. This is similar to what is achievable with physical transfer standards in an expert laboratory but with a much more tedious and time-consuming procedure and severe geometry restrictions $[8,14,17,19]$.

\section{Conclusion and outlook}

With the Calibration Kit Spectral Fluorescence Standards BAM-F001-BAM-F005, we developed a simple, flexible, and traceable tool for the characterization and long-term control of the relative spectral responsivity of fluorescence instruments as well as for the determination of corrected, i.e., instrument-independent emission data. This provides the basis for an improved reliability and comparability of emission data across instruments and over time on a very broad level and presents a first step towards the standardization of fluorescence techniques. This kit that has been optimized for spectrofluorometers, a measurement geometry of $0^{\circ} / 90^{\circ}$, and $1-\mathrm{cm}$ cells, can be employed for a broad variety of measurement geometries and different formats or sample containers. Due to the unique properties of its components, BAM-F001BAM-F005 can be adapted to the calibration of other types of fluorescence instruments such as e.g., microplate readers and spectral imaging systems with proper consideration of the underlying measurement principles. The potential and limitations of the kit for the characterization of the latter are currently being investigated.

Acknowledgments Financial support from the German Ministry of Economics and Labor (BMWA) is gratefully acknowledged (grants
BMWA VI A2-18 and VI A2-17/03). We express our gratitude to Dr J. Hollandt and Dr R. D. Taubert from PTB for the calibration of the spectral radiance transfer standards and provision of the spectral radiance of the tungsten ribbon lamp displayed in Fig. 2, to Dr A. Höpe from PTB for the calibration of the diffuse reflectance standard, and to Dr B. Schönenberger and Dipl.-Biol. P. Nording from SigmaAldrich $\mathrm{GmbH}$ (former Fluka Production $\mathrm{GmbH}$ ) for the supply of the kit dyes and the solvent. We are also very grateful for the analytical characterization of the kit dyes by our BAM colleagues Dr U. Erhardt, Dr P. Lehnink-Habrink, Dipl.-Chem. O. Hahnebeck, and Mrs R. Schmieder (chromatography), Dr M. Koch and Mrs E. Tzscheutschler (water content, Karl Fischer titration), and Dr F. Malz (quantitative NMR) as well as to Dr W. Bremser for help with the determination of the uncertainty budget for the corrected emission spectra of the kit components.

\section{References}

1. Lakowicz JR (1999) Principles of fluorescence spectroscopy, 2nd edn. Kluwer Academic/Plenum, New York

2. Lakowicz JR (ed) (1992-2004) Topics in fluorescence spectroscopy, series vol 1-8. Plenum, New York

3. Wolfbeis OS (series ed) (2001-2004) Springer series on fluorescence, methods and applications, vol 1-3. Springer, Berlin

4. Schulman SG (ed) (1985-1993) Molecular luminescence spectroscopy parts 1-3. Wiley Interscience, New York

5. Mason WT (1999) Fluorescent and luminescent probes for biological activity, 2nd edn. Academic, San Diego

6. Resch-Genger U, Hoffmann K, Nietfeld W, Engel A, Neukammer J, Nitschke R, Ebert B, Macdonald R (2005) How to improve quality assurance in fluorometry: fluorescence-inherent sources of error and suited fluorescence standards. J Fluoresc 15(3):337-362 and references therein

7. Monte C, Resch-Genger U, Pfeifer D, Taubert RD, Hollandt J (2005) Linking fluorescences measurements to radiometric units, Metrologia (in press)

8. Resch-Genger U, Pfeifer D, Monte C, Pilz W, Hoffmann A, Spieles M, Rurack K, Hollandt J, Taubert D, Schönenberger B, Nording P (2005) Traceability in fluorometry: part II. Spectral fluorescence standards. J Fluoresc 15(3):315-336

9. Certificate of the Federal Institute for Materials Research and Testing (BAM) (2006) Certified Reference Materials BAM-F001, BAM-F002, BAM-F003, BAM-F004, and BAM-F005, Calibration Kit Spectral Fluorescence Standards, for the determination of the relative spectral responsivity of fluorescence instruments, January 10,2006

10. Hollandt J, Taubert DR, Seidel J, Resch-Genger U, GuggHelminger A, Pfeifer D Monte C (2005) Traceability in fluorometry: part I physical standards. J Fluoresc 15(3):301-313

11. Eastman JW (1966) Standardization of fluorescence spectra and the calibration of spectrofluorimeters. Appl Opt 5(7):11251132

12. Miller JN (1981) Standards in fluorescence spectrometry. Ultraviolett Spectrometry Group, London

13. Velapoldi RA, Tonnesen HH (2004) Corrected emission spectra and quantum yields for a series of fluorescent compounds in the visible spectral region. J Fluoresc 14(4):465-472

14. Hofstraat JW, Latuhihin MJ (1994) Correction of fluorescencespectra. Appl Spectrom 48(4):436-447

15. Hoffmann K, Monte C, Pfeifer D, Resch-Genger U (2005) Standards in fluorescence spectroscopy: simple tools for the characterization of fluorescence instruments. GIT Lab J (5):2-4

16. Velapoldi RA, Mielenz KD (1980) A fluorescence standard reference material: quinine sulfate dihydrate. NBS Special Publication, PB 80132046, Springfield, VA, pp 260-264 
17. Gardecki JA, Maroncelli M (1998) Set of secondary emission standards for calibration of the spectral responsivity in emission spectroscopy. Appl Spectrom 52(9):1179-1189

18. Hoffmann K, Resch-Genger U, Nitschke R (2005) Simple Tool for the Standardization of Confocal Spectral Imaging Systems. GIT Imaging Microsc (3):18-19
19. Bremser W, de Rose P, Duewer DS, Ebert B, Hoffmann A, Hollandt J, Macdonald R, Monte C, Pfeifer D, Resch-Genger U, Taubert DR, Zwinkels $\mathrm{J}$ (manuscript in preparation)

20. Pfeifer D, Hoffmann A, Monte C, Spieles M, Bremser W, ReschGenger $\mathrm{U}$ (manuscript in preparation 